\title{
REVISTA
}

Revista Educación

ISSN: 0379-7082

ISSN: 2215-2644

revedu@gmail.com

Universidad de Costa Rica

Costa Rica

\section{Aprendizaje estadístico basado en niveles de investigación}

Alonso Trujillo, Javier; Alonso Ricardez, Abraham; Valera Mota, Myrna Miriam; Cuevas Guajardo, Leticia Aprendizaje estadístico basado en niveles de investigación

Revista Educación, vol. 46, núm. 1, 2022

Universidad de Costa Rica, Costa Rica

Disponible en: https://www.redalyc.org/articulo.oa?id=44068165037

DOI: https://doi.org/10.15517/revedu.v46i1.45425

\section{(c) $(1) \Theta$}

Esta obra está bajo una Licencia Creative Commons Atribución-NoComercial-SinDerivar 3.0 Internacional. 
Artículos científicos, experiencia de acción social o didáctica

\section{Aprendizaje estadístico basado en niveles de investigación}

Statistical Learning Based on Research Levels

Javier Alonso Trujillo

Universidad Nacional Autónoma de México, México

alonsot1212@yahoo.com.mx

DOI: https://doi.org/10.15517/revedu.v46i1.45425 Redalyc: https://www.redalyc.org/articulo.oa?

(DD https://orcid.org/0000-0002-4257-3718

Abraham Alonso Ricardez

Universidad Nacional Autónoma de México, México

abraham_alonso@iztacala.unam.mx

(iD https://orcid.org/0000-0002-0983-9836

Myrna Miriam Valera Mota

Universidad Nacional Autónoma de México, México

valeramota@gmail.com

iD https://orcid.org/0000-0002-8966-7957

Leticia Cuevas Guajardo

Universidad Nacional Autónoma de México, México

leticiacuevas1@hotmail.com

(iD https://orcid.org/0000-0002-0253-4909

Recepción: 22 Febrero 2021

Aprobación: 12 Julio 2021

\section{Resumen:}

En este artículo se presenta una estrategia didáctica, cuyo propósito fue mejorar el aprendizaje de la Estadística en estudiantes de la licenciatura en Enfermería en una institución educativa de nivel superior en México. Lo particular de la estrategia fue que se apoyó en los niveles de investigación que fueron propuestos en la Master Class de Bioestadística, organizada por la Sociedad Hispana de Investigadores Científicos. El objetivo del estudio fue demostrar que el aprendizaje estadístico se incrementa con la aplicación de la estrategia didáctica. Con un diseño cuasi-experimental, con cuatro exámenes del aprendizaje, se compararon los resultados de los exámenes con un análisis multivariante intrasujetos. 33 personas estudiantes fueron seleccionadas según el criterio de investigación, cuyo origen fue una población de 135 estudiantes. Los resultados mostraron la evolución del aprendizaje estadístico durante el semestre escolar, en el que se destaca un incremento raquítico en la calificación media en los primeros tres exámenes (4.3, 5.6 y 6.3 respectivamente), para después volver a disminuir hasta 5.6 de calificación media en el cuarto examen. Se concluye que, no obstante el escaso mejoramiento del aprendizaje, hubo casos particulares en los que se obtuvieron hasta 8.8 puntos en la escala de 0 a 10, lo que permite sugerir que el éxito de la estrategia podría estar asociado a factores personales específicos.

Palabras Clave: Aprendizaje activo, Enseñanza superior, Enseñanza de la Estadística, Estadística, Estrategias educativas, Estudiante universitario, Evaluación comparativa, Evaluación de la educación.

\section{Abstract:}

This study examines a Biostatistics education strategy among undergraduate nursing students at a university in Mexico. This strategy is unique in that it relies on research proposed by the Biostatistics Master Class organized by the Hispanic Society of Scientific Researchers. The objective of the study was to demonstrate how this teaching strategy is useful in improving Biostatistics education. This study used a quasi-experimental design and four exams to verify student knowledge. Exam results were compared applying a multivariate within-subject analysis. A select group of 33 students were chosen from 135 students, based on the criteria of the researchers. Results reveal a rise in statistical learning among students during the school semester, highlighting a slight increase in average grades after the three exams (4.3, 5.6 and 6.3 respectively) which progressively dropped to 5.6 - the average grade for the fourth exam. It was concluded that, despite limited improvement in student performance, there were particular cases in 
which up to 8.8 points were obtained on the scale from 0 to 10 , which suggests that the success of the strategy could be associated with specific individual factors.

KEYWORDS: Active Learning, Higher Education, Teaching Statistics, Statistics, Educational Strategies, University Student, Benchmarking, Educational Evaluation.

\section{INTRODUCCIÓN}

En este trabajo, se plantea la problemática relacionada con el aprendizaje de procedimientos estadísticos en estudiantes de nivel universitario y sus posibles orígenes. Se puntualizan situaciones específicas para la población estudiantil de Enfermería y pone a prueba una estrategia didáctica para mejorar el aprendizaje.

En México, el $35 \%$ del estudiantado a nivel secundaria carece de un nivel mínimo de competencia en lectura, matemáticas y ciencias, es decir, no alcanzan a obtener calificaciones consideradas aprobatorias; solo el $1 \%$ del estudiantado obtuvo un desempeño en los niveles de competencia más altos en al menos un área, según fue reportado por el Programa para la Evaluación Internacional de Alumnos (PISA, por sus siglas en inglés) en 2018 (Salinas et al., 2018). Estas deficiencias probablemente continuarán reproduciéndose durante la formación académica en el nivel medio superior y superior.

En este trabajo se abordó el tema de aprendizaje estadístico con la intención de encontrar algunos indicios de la dificultad que representa para el estudiantado universitario. La justificación para desarrollar este trabajo radica en que la investigación de la enseñanza de la estadística aún se encuentra en sus inicios. Obras como la de Batanero (2001) sugieren que la didáctica de la Estadística será tema de estudio en este siglo XXI, en virtud de la necesidad que existe para formar una ciudadanía estadísticamente culta. La proliferación de organizaciones, publicaciones y tecnología estadística constituirán el sustrato básico de la didáctica de la Estadística. Los conceptos estadísticos y la interpretación de ellos en diferentes circunstancias es una problemática que todavía está siendo abordada por la epistemología estadística. El fenómeno de la aleatoriedad, el concepto de probabilidad y los razonamientos teóricos que hay que hacer para comprender un procedimiento estadístico es solo la punta del iceberg dentro de la problemática en la enseñanza de la estadística.

Es probable que la estrecha relación que guardan las Matemáticas y la Ciencia con la Estadística contribuya a crear una barrera mental en el estudiantado y a que su aprendizaje se vuelva difícil y problemático. Algunos de los argumentos que se señalan son los siguientes: .si yo voy a ser contador o ingeniero, ¿para qué quiero Estadística?"; también, en otras carreras como Enfermería o Medicina se han reportado dichos como .La tenemos que estudiar solo para poder titularnos. (Ferreri et al., 1999, p.117).

Se han reportado dificultades en el aprendizaje de la Estadística en estudiantes de Medicina (Hernández et al., 2012). Asimismo, se han observado dificultades estudiantes de Ingeniería para comprender el significado de conceptos estadísticos y la relación que guardan entre ellos (Maris y Aznar, 2018). Al respecto, personas investigadoras de Noruega (Hilde et al., 2021) afirman que, en el estudiantado universitario, existen perfiles cognitivos y de personalidad que a veces no son compatibles con ciertos tipos de estrategias de enseñanza en Estadística. Por otra parte, se han documentado diversas experiencias docentes que señalan las dificultades que tiene el estudiantado para comprender conceptos inherentes a la Estadística. Algunas personas autoras han señalado que, durante su práctica docente, la asignatura Estadística, es una especie de dolor de cabeza para el alumnado, tanto en el pregrado como en el posgrado, así surge que esta problemática podría tener sus raíces en la comprensión de conceptos asociados a fórmulas matemáticas (Chance, et al., 2004; Inzunza y Jiménez, 2013; Pulido, 2009). Al respecto, Medina (2014) señala que las competencias cognitivas y procedimentales en investigación científica de estudiantes de Enfermería son deficientes, pues en dos evaluaciones que realizó la autora se obtuvo puntajes medios de $5.7 \pm 1.1$ y $4.9 \pm 1.5$, respectivamente. También se han reportado actitudes de rechazo hacia la Estadística en estudiantes de Enfermería, esto debido a diversos factores, entre ellos: indiferencia hacia la Estadística, el estudiantado no vislumbra su aplicación profesional; escaso 
dominio del profesorado respecto a los contenidos; el presupuesto no alcanza para comprar licencias de software estadístico; y una orientación predominantemente asistencial planteada en el perfil de egreso del estudiantado (Alonso et al., 2017).

En virtud de lo anterior, es evidente que el estudiantado de la carrera de Enfermería no está exento de esta problemática, y es indispensable que aprenda y comprenda conceptos metodológicos y estadísticos para desarrollar competencias investigativas (Alonso et al., 2018). Recientemente, Alonso et al. (2020) analizaron los resultados estadísticos de 198 reportes de investigación de estudiantes de Enfermería, donde observaron que solo 40 trabajos plantearon el uso de pruebas de hipótesis; 96 trabajos no reportaron uso de pruebas de hipótesis, pero hay indicios de que las usaron; 94 trabajos no interpretaron el p-valor, cuando debieron haberlo hecho; 11 trabajos lo interpretaron incorrectamente y solo 31 trabajos lo interpretaron correctamente.

La problemática descrita motivó a escribir este artículo, en el que se presenta una estrategia didáctica con propósito de mejorar el aprendizaje de la Estadística en estudiantes de la licenciatura en Enfermería en una institución educativa de nivel superior en México. Lo particular de la estrategia es que se apoya en los niveles de investigación que fueron propuestos en la Máster-Class de Bioestadística organizada por la Sociedad Hispana de Investigadores Científicos.

\section{ESTRATEGIA APRENDIZAJE BASADO EN NIVELES DE INVESTIGACIÓN}

Los niveles de investigación han sido propuestos por Supo (2014) en los seminarios que ha impartido en las denominadas Máster-Class de Bioestadística. Estas son reuniones anuales que se realizaron en diversas ciudades latinoamericanas y que tuvieron como propósito reflexionar acerca de estrategias didácticas para mejorar la enseñanza de la metodología de la investigación científica, así como identificar algunos fundamentos didácticos que apoyan esas estrategias. Por ejemplo, el aprendizaje experiencial, la enseñanza presencial o el constructivismo. En este trabajo se ha retomado la propuesta de Supo (2014), pero orientada a mejorar la enseñanza de la Estadística. Por esta razón, se sometió a prueba la estrategia y se evaluó su resultado como un intento por resolver la problemática identificada en el contexto escolar. La estrategia considera la enseñanza presencial, el aprendizaje basado en problemas (utilizando datos contextualizados a la profesión de Enfermería), el aprendizaje experiencial y el trabajo en equipo.

Nivel exploratorio. La estrategia inicia con el análisis de algunos métodos de investigación que se aplican en los estudios con enfoque cualitativo. En este sentido, se trata de que el estudiantado conozca las características generales del enfoque cualitativo, el cual, por cierto, no hace uso de la estadística, pero que - gracias a algunos métodos cualitativos como la fenomenología, la hermenéutica y el construct ivismo- permiten, de manera heurística, construir una definición conceptual del objeto de estudio y dar origen a la variable de interés que se estudiará a través del resto de los niveles de investigación.

Nivel descriptivo. Se refiere, como su nombre lo indica, a realizar procedimientos estadísticos de orden descriptivo; se aplican conceptos como organización de datos, tipo de variables, escalas de medición, elaboración de gráficos y tablas, cálculo de medidas de tendencia central, variabilidad y posición. Por último se incluyen cálculos de los intervalos de confianza al $95 \%$. Este nivel se caracteriza por incluir análisis estadísticos de tipo univariado.

Nivel relacional. La relación bivariada es la principal característica de los procedimientos estadísticos que se aprenden en este nivel. Por ejemplo, se estudian las correlaciones entre variables numéricas y las asociaciones entre variables categóricas. Se introduce la comparación estadística entre grupos independientes, en la que el estudiantado aprende a diseñar proyectos con el uso de una variable fija y otra aleatoria. El alumnado aprende a realizar pruebas de hipótesis acompañadas de los coeficientes que determinan la fuerza de correlación o de asociación entre variables. 
Nivel explicativo. La característica de este nivel es el análisis multivariado, representado, en su forma más simple, por la incorporación simultánea de tres variables incluidas en problemas con datos contextualizados a la práctica profesional de la Enfermería. Aquí, se utilizan diseños experimentales, en cualquiera de sus modalidades (experimentales puros, de bloques completamente aleatorios, cuasi-experimentales, diseños factoriales, etc.) Los experimentos promueven el aprendizaje basado en problemas y, como se trabaja en equipo, se fomenta la colaboración constructivista en el estudiantado.

\section{Fundamento TEÓRico de la ESTRATEgia.}

El aprendizaje experiencial. Desde la perspectiva de Díaz Barriga, la idea de que el currículo y la enseñanza deben centrarse en las necesidades, intereses y experiencias del alumnado tomó sentido a principios del siglo $\mathrm{XX}$. Por ello, se necesita que las escuelas enseñen al estudiantado a desarrollar estrategias para aprender haciendo y así construir su propio conocimiento vinculado a situaciones reales de su futura práctica profesional. La estrategia se fundamenta en lo que se ha llamado Aprendizaje Experiencial, entendido como aquel paradigma en el que la escuela es una institución social en la que puede desarrollarse una vida comunitaria que constituye el soporte de la educación. Posner sugiere que el aprendizaje experiencial se basa en el supuesto de que todo lo que le ocurre al estudiantado influye en su vida (Díaz-Barriga, 2006; Posner, 1993).

El constructivismo. Ortiz (2015) plantea que existe una interacción entre el profesorado y el alumnado, un intercambio dialéctico entre los conocimientos de las dos partes, de tal forma que se puede llegar a una síntesis productiva para ambas partes y, en consecuencia, los contenidos estudiados permiten lograr un aprendizaje significativo. La vida comunitaria se da en torno a la cooperación social y de la participación democrática de las actividades sociales del grupo; esto sugiere la autodisciplina a partir del compromiso en una tarea constructiva y con base en la propia decisión individual. El aprendizaje se irá construyendo por cada persona, disciplinada, comprometida, consciente de la necesidad de vincular poco a poco los conocimientos adquiridos en cada nivel investigativo (Figura 1).

Enseñanza presencial. Considera la participación del profesorado como el orador principal durante la clase. A pesar de que se han señalado particularidades en la enseñanza que podrían perjudicar al estudiantado, por ejemplo, propiciar alumnado con actitudes pasivas, sigue siendo parte del desempeño académico en la mayoría de las materias, de las carreras y de las escuelas. El estudiantado sigue confiando en lo que el profesorado le enseña. La enseñanza presencial, también conocida como enseñanza tradicional, consiste en una instrucción en persona y que no requiere ningún componente en línea; el grupo se reúne en el aula, con contenido entregado oralmente y por escrito (Orosz, 2016).

Aprendizaje basado en problemas. Es una metodología centrada en el aprendizaje, en la investigación y reflexión que sigue el alumnado para llegar a una solución ante un problema planteado por su profesor o profesora. Según Prieto (2006) representa una estrategia eficaz y flexible que, a partir de lo que hace el alumnado, puede mejorar la calidad de su aprendizaje universitario en aspectos muy diversos. 


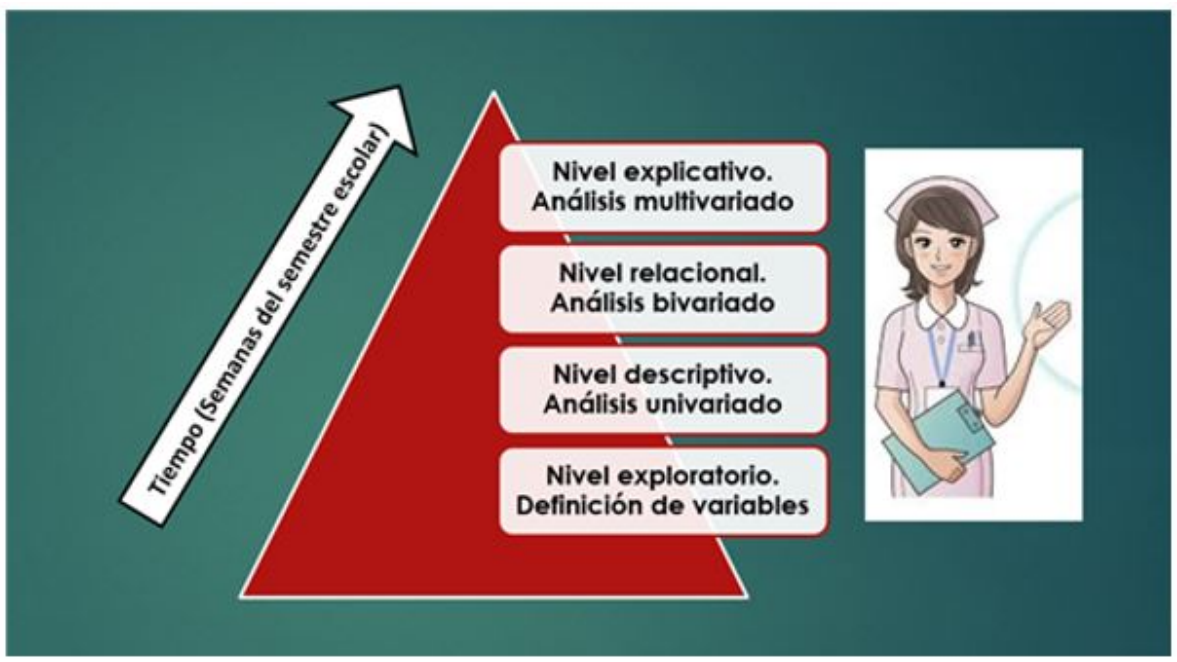

FIGURA 1.

Estrategia aprendizaje basado en niveles de investigación

Nota. La construcción del conocimiento es un proceso que se logra durante el semestre, poco a poco, se inicia con lo simple hasta llegar a lo complejo. El constructivismo, el aprendizaje basado en problemas, el aprendizaje experiencial y la enseñanza presencial (tradicional), son pilares de la estrategia propuesta Fuente: elaboración propia.

\section{Complementos de la ESTRATEgia.}

Aunque la enseñanza de la estadística se fundamenta en los niveles de investigación, es necesario conocer los complementos didácticos de esta.

1. Libro de ejercicios para cada nivel investigativo. Este recurso de apoyo incluye 32 ejercicios con planteamientos contextuales (problemas de investigación) posibles de ocurrir en la práctica de la Enfermería. El libro se titula Investigación cientifica en Enfermería. Análisis de datos (Alonso y Alonso, 2019).

2. Clases magistrales con énfasis en teoría estadística. Una explicación de la Teoría de Fisher y de Neyman-Pearson sirve como punto de apoyo inicial al resto de los contenidos. Durante la enseñanza presencial, se describen y explican conceptos estadísticos según el nivel de investigación en el que se apliquen y los métodos cuantitativos propios de cada nivel. Por citar solo algunos ejemplos, en el nivel descriptivo se estudian los siguientes conceptos: tipos de variables, escalas de medición, estimadores puntuales, medidas de tendencia central y variabilidad y su expresión a través de diversos tipos de gráficos. Los análisis estadísticos son univariados. En el nivel relacional se estudian los conceptos: población, muestra, parámetro, estadístico, intervalos de confianza al $95 \%$, nivel de significancia, valor p, distribución normal, área de rechazo, área de no rechazo, chi cuadrada de bondad de ajuste, chi cuadrada de independencia, chi cuadrado de McNemar, chi cuadrada de homogeneidad, prueba de correlación de Pearson, prueba de correlación Rho de Spearman. Los análisis estadísticos son bivariados. Por último, en el nivel explicativo se integran los conceptos de los niveles anteriores para aplicarlos en pruebas de análisis multivariado, como Chi Cuadrado de Mantel-Haenszel, t de Student para grupos independientes, t de Student para muestras relacionadas y pruebas de normalidad.

3. Laboratorio de computación. Se hace uso de este recurso 96 horas durante el semestre, lo cual corresponde al $50 \%$ del tiempo curricular en el cual se hace uso intensivo de software estadístico, fundamentalmente Microsoft Excel 2013 e IBM SPSS Statistics versión 22. 
4. Proyecto de investigación. El alumnado construye e implementa un proyecto de investigación, el cual se trabaja en equipo y le brinda la oportunidad de aplicar sus conocimientos teóricos y prácticos. Se destinan 48 horas durante el semestre, lo cual corresponde al $25 \%$ del tiempo curricular.

Se considera importante citar al Consejo Nacional de Maestros de Matemáticas (NCTM por sus siglas en inglés), el cual se pronuncia en favor de que la responsabilidad de modificar planes de estudios y ampliar tiempos para los módulos no recaiga en el profesorado; sin embargo, apoyarse en el diseño de estrategias para mejorar el aprendizaje que plantea cada docente, podría solucionar algunos de los problemas asociados con el aprendizaje del estudiantado (National Council of Teachers of Mathematics [NCTM], 2014).

Hipótesis de investigación. La autoría de este trabajo plantea que la estrategia educativa Aprendizaje basado en niveles de investigación, incrementa el aprendizaje estadístico en el estudiantado de Enfermería.

Con base en lo anterior, el objetivo de este trabajo fue demostrar con evidencias estadísticas que el aprendizaje estadístico se incrementa conforme avanza el semestre escolar como efecto de la estrategia educativa.

\section{Materiales Y MÉTODOS}

Tipo de investigación cuasi-experimental, longitudinal ( 4 mediciones), prospectiva y analítica. Por el enfoque multivariado, se ubica en el nivel de investigación explicativo. El diseño de la investigación consistió en un análisis de varianza con un factor intrasujetos. La variable aleatoria fue el aprendizaje estadístico, mientras que la variable fija fueron las cuatro mediciones realizadas, a lo que se denominó exámenes por nivel investigativo. El grupo experimental estuvo integrado por treinta y tres estudiantes de séptimo semestre 2020-1 de la licenciatura en Enfermería, que recibieron durante un semestre escolar (agosto a diciembre de 2019) la estrategia educativa denominada Aprendizaje basado en niveles de investigación, descrita ampliamente en párrafos anteriores.

Población. De acuerdo con la línea de investigación que se desarrolla en este trabajo, se definió a la población como aquel conjunto de estudiantes de Enfermería que cursa el módulo Métodos Cuantitativos de Investigación en el séptimo semestre de la licenciatura en Enfermería, en una institución educativa de nivel superior en México. En el semestre en el que se desarrolló esta investigación, la población estuvo integrada por 135 estudiantes; cabe señalar que cada semestre se incrementa aproximadamente entre un $5 \%$ y $10 \%$. En virtud de que la población total resulta inaccesible a los fines de esta investigación, principalmente porque se aplican otras estrategias de enseñanza, se obtuvo una muestra no probabilística. Tamaño de la muestra. Se eligió a treinta y tres personas estudiantes inscritas formalmente en el módulo de métodos cuantitativos de investigación, específicamente en el grupo 2701. Técnica de muestreo. Se aplicó la técnica de muestreo conocida como muestreo según criterio, la cual consistió en elegir a las unidades de estudio que, a criterio del grupo investigador, cumplían con una serie de características académicas propicias para la medición de las variables de estudio. Obviamente una de esas características (y que no tenía ningún otro grupo), fue que se tuviera control didáctico y evaluativo, es decir, que se les aplicara la estrategia y se pudiera evaluar.

Instrumento de medición. Se elaboraron cuatro exámenes con carácter formal respecto a la evaluación del estudiantado y su respectiva acreditación. En cada examen se incluyeron y midieron contenidos teóricos y prácticos específicos de cada uno de los niveles de investigación planteados en la Figura 1. A estos instrumentos se les denominó: examen 1, examen 2, examen 3 y examen 4.

Cada examen, de acuerdo con el Reglamento General de Exámenes de la Institución Educativa (Universidad Nacional Autónoma de México, 1997), solo puede alcanzar valores correspondientes a la escala que va desde 0 hasta 10 puntos. Para considerar aprobada a una persona estudiante, la calificación mínima debe de ser 6 puntos. 
Validez interna del instrumento. La validez de contenido consistió en que todos los reactivos estuvieron fundamentados en contenidos relacionados con procedimientos estadísticos asociados a los niveles de investigación, los cuales están incluidos en el programa del módulo Métodos Cuantitativos de Investigación (Universidad Nacional Autónoma de México, 2015). Adicionalmente, se realizaron correlaciones bivariadas entre las calificaciones de cada uno de los exámenes y el promedio final, con el que se obtuvieron coeficientes de correlación directos, fuertes y significativos, salvo en el examen 3, en el cual la correlación fue directa, moderada y significativa.

Las fases de recolección de los datos fueron cuatro. Aproximadamente, cada mes se aplicaba cada uno de los exámenes (agosto-septiembre-octubre-noviembre), una vez que en el aula ya se habían estudiado las características de cada nivel de investigación y se habían realizado los ejercicios correspondientes. Cabe señalar que también se desarrolló, paralelamente al estudio de los niveles de investigación, un proyecto de investigación.

Plan de análisis estadístico. Se calcularon medidas de tendencia central y de variabilidad. Se obtuvieron los intervalos de confianza al 95 \%. Se demostró la normalidad de los datos (Prueba Kolmogorov-Smirnov) y el equivalente a homocedasticidad de los datos (esfericidad con la Prueba W de Mauchly). El análisis de la varianza (ANOVA), con un factor intrasujetos, también llamado contraste multivariante, se realizó con el estadístico de prueba F de Fisher, al explorar cuatro algoritmos: Traza de Pillai, Lambda de Wilks, Traza de Hotelling y Raíz mayor de Roy. El nivel de significancia aplicado a las pruebas de hipótesis fue $\leq 0.05$. Se utilizó el software estadístico Microsoft Excel 2013, así como IBM SPSS Statistics versión 22.

Tipo de investigación cuasi-experimental, longitudinal ( 4 mediciones), prospectiva y analítica. Por el enfoque multivariado, se ubica en el nivel de investigación explicativo. El diseño de la investigación consistió en un análisis de varianza con un factor intrasujetos. La variable aleatoria fue el aprendizaje estadístico, mientras que la variable fija fueron las cuatro mediciones realizadas, a lo que se denominó exámenes por nivel investigativo. El grupo experimental estuvo integrado por treinta y tres estudiantes de séptimo semestre 2020-1 de la licenciatura en Enfermería, que recibieron durante un semestre escolar (agosto a diciembre de 2019) la estrategia educativa denominada Aprendizaje basado en niveles de investigación, descrita ampliamente en párrafos anteriores.

Población. De acuerdo con la línea de investigación que se desarrolla en este trabajo, se definió a la población como aquel conjunto de estudiantes de Enfermería que cursa el módulo Métodos Cuantitativos de Investigación en el séptimo semestre de la licenciatura en Enfermería, en una institución educativa de nivel superior en México. En el semestre en el que se desarrolló esta investigación, la población estuvo integrada por 135 estudiantes; cabe señalar que cada semestre se incrementa aproximadamente entre un $5 \%$ y $10 \%$. En virtud de que la población total resulta inaccesible a los fines de esta investigación, principalmente porque se aplican otras estrategias de enseñanza, se obtuvo una muestra no probabilística. Tamaño de la muestra. Se eligió a treinta y tres personas estudiantes inscritas formalmente en el módulo de métodos cuantitativos de investigación, específicamente en el grupo 2701. Técnica de muestreo. Se aplicó la técnica de muestreo conocida como muestreo según criterio, la cual consistió en elegir a las unidades de estudio que, a criterio del grupo investigador, cumplían con una serie de características académicas propicias para la medición de las variables de estudio. Obviamente una de esas características (y que no tenía ningún otro grupo), fue que se tuviera control didáctico y evaluativo, es decir, que se les aplicara la estrategia y se pudiera evaluar.

Instrumento de medición. Se elaboraron cuatro exámenes con carácter formal respecto a la evaluación del estudiantado y su respectiva acreditación. En cada examen se incluyeron y midieron contenidos teóricos y prácticos específicos de cada uno de los niveles de investigación planteados en la Figura 1. A estos instrumentos se les denominó: examen 1, examen 2, examen 3 y examen 4.

Cada examen, de acuerdo con el Reglamento General de Exámenes de la Institución Educativa (Universidad Nacional Autónoma de México, 1997), solo puede alcanzar valores correspondientes a la escala 
que va desde 0 hasta 10 puntos. Para considerar aprobada a una persona estudiante, la calificación mínima debe de ser 6 puntos.

Validez interna del instrumento. La validez de contenido consistió en que todos los reactivos estuvieron fundamentados en contenidos relacionados con procedimientos estadísticos asociados a los niveles de investigación, los cuales están incluidos en el programa del módulo Métodos Cuantitativos de Investigación (Universidad Nacional Autónoma de México, 2015). Adicionalmente, se realizaron correlaciones bivariadas entre las calificaciones de cada uno de los exámenes y el promedio final, con el que se obtuvieron coeficientes de correlación directos, fuertes y significativos, salvo en el examen 3, en el cual la correlación fue directa, moderada y significativa.

Las fases de recolección de los datos fueron cuatro. Aproximadamente, cada mes se aplicaba cada uno de los exámenes (agosto-septiembre-octubre-noviembre), una vez que en el aula ya se habían estudiado las características de cada nivel de investigación y se habían realizado los ejercicios correspondientes. Cabe señalar que también se desarrolló, paralelamente al estudio de los niveles de investigación, un proyecto de investigación.

Plan de análisis estadístico. Se calcularon medidas de tendencia central y de variabilidad. Se obtuvieron los intervalos de confianza al 95 \%. Se demostró la normalidad de los datos (Prueba Kolmogorov-Smirnov) y el equivalente a homocedasticidad de los datos (esfericidad con la Prueba W de Mauchly). El análisis de la varianza (ANOVA), con un factor intrasujetos, también llamado contraste multivariante, se realizó con el estadístico de prueba F de Fisher, al explorar cuatro algoritmos: Traza de Pillai, Lambda de Wilks, Traza de Hotelling y Raíz mayor de Roy. El nivel de significancia aplicado a las pruebas de hipótesis fue $\leq 0.05$. Se utilizó el software estadístico Microsoft Excel 2013, así como IBM SPSS Statistics versión 22.

\section{Resultados}

Algunos de los aspectos considerados cuando se planeó la estrategia educativa que aquí se presenta, fueron que esta se incorporara al proceso de enseñanza-aprendizaje, que fuera coherente con el programa de la materia y que pueda medirse su efecto. Así, en esta sección del trabajo se presentan los resultados obtenidos en los exámenes de evaluación aplicados.

Estrategia descriptiva. En la Tabla 1 se observan los estadísticos descriptivos obtenidos luego de la aplicación de los cuatro exámenes al grupo de estudiantes. De manera general, cada examen expresa el aprendizaje estadístico de cada nivel investigativo, es decir, en el examen 1 se evaluaron contenidos teórico-prácticos relativos al nivel exploratorio, en el examen 2 lo relativo al nivel descriptivo y así sucesivamente (Véase Figura 1). Si se fija la atención en el promedio obtenido por el grupo en cada examen, se notará una tendencia hacia un mejoramiento del aprendizaje estadístico, al menos en las tres primeras mediciones. En la cuarta medición ocurre un decremento del aprendizaje, sin embargo, las calificaciones medias son reprobatorias, a excepción de las del examen 3, en el que se obtuvo un promedio de 6.3 puntos. El lector se deberá fijar en la variabilidad que mostró cada medición y, sobre todo, deberá observar el valor mínimo y el máximo obtenido por el estudiantado. El rango observado expresa evaluaciones extremas en todos los casos. Llaman la atención algunos casos con calificaciones de 0 o 1.9 puntos, en contraste con sujetos que obtuvieron calificaciones aprobatorias con un máximo de 8.8 puntos. Es decir, hubo alumnado con nulo aprendizaje estadístico, así como también lo hubo con aprendizajes aceptables o buenos, sin llegar a ser excelentes. 
TABLA 1

Estadísticos descriptivos obtenidos en cada una de las mediciones

\begin{tabular}{|c|c|c|c|c|}
\hline & Examen 1 & Examen 2 & Examen 3 & Examen 4 \\
\hline Válido & 33 & 33 & 33 & 33 \\
\hline Perdidos & 0 & 0 & 0 & 0 \\
\hline Media & 4.3 & 5.6 & 6.3 & 5.6 \\
\hline $\begin{array}{l}\text { Error estándar de la } \\
\text { media }\end{array}$ & 0.3 & 0.2 & 0.4 & 0.4 \\
\hline Mediana & 4.3 & 5.6 & 7.0 & 6.0 \\
\hline Desviación estándar & 1.6 & 1.4 & 2.0 & 2.1 \\
\hline Varianza & 2.6 & 1.8 & 4.2 & 4.3 \\
\hline Rango & 7.1 & 5.4 & 8.8 & 8.7 \\
\hline Mínimo & 0.0 & 1.9 & 0.0 & 0.0 \\
\hline Máximo & 7.1 & 7.3 & 8.8 & 8.7 \\
\hline
\end{tabular}

Fuente: elaboración propia.

Nota: resultados obtenidos a partir de datos de campo.

Intervalos de confianza. La variabilidad estadística para los datos registrados se observa en la Tabla 2, en la que se observan los valores de los intervalos de confianza. Desde una perspectiva teórica, sin inferir nada a la población de origen, el $95 \%$ del estudiantado participante podría tener aprendizajes estadísticos no aprobatorios en los exámenes 1, 2 y 4 . Solo en el examen 3, que corresponde a contenidos del nivel relacional (procedimientos estadísticos bivariados), se alcanzó una calificación media aprobatoria. Estadísticamente, es probable encontrar estudiantes con calificaciones muy bajas, pero no de cero. Asimismo, es probable encontrar estudiantes con calificaciones excelentes. Esto permite reflexionar acerca de los factores que influyen en el rendimiento académico y tratar de explicar las bajas calificaciones medias. Según Garbanzo (2007), entre los determinantes que influyen en el rendimiento académico están los personales, los sociales y los institucionales.

TABLA 2

Intervalos de confianza al $95 \%$ para los datos

Examen 1 Examen 2 Examen 3 Examen 4

\begin{tabular}{lllll}
\hline Media & 4.3 & 5.6 & 6.3 & 5.6 \\
\hline Desviación estándar & 1.6 & 1.4 & 2.0 & 2.1 \\
\hline IC 95\% Inferior & 1.2 & 2.9 & 2.3 & 1.5 \\
\hline IC 95\% Superior & 7.5 & 8.2 & 10.0 & 9.6 \\
\hline
\end{tabular}

Fuente: elaboración propia.

Nota: resultados obtenidos a partir de datos de campo.

Análisis multivariante intrasujetos (ANOVA intrasujetos). Una forma de evaluar el efecto de la estrategia educativa sobre los resultados obtenidos en cada examen, es la realización de un análisis comparativo intrasujetos o también llamado multivariante por la naturaleza de las mediciones.

En la Tabla 3, se aprecia que el software estadístico ejecutó cuatro algoritmos multivariantes que arrojaron el valor del estadístico de prueba y el p-valor. En este último caso, la hipótesis que es más probable que sea cierta es la que señala que existen diferencias significativas entre las mediciones de los cuatro exámenes. Cabe señalar que, por tratarse de un análisis intrasujetos, no existe factor de análisis intragrupos, por lo que no es posible demostrar la homogeneidad de las varianzas, pero, en su lugar, se ejecutó la prueba de esfericidad W de Mauchly, la cual, junto con la demostración de normalidad, permite afirmar que existen los supuestos teóricos para aceptar la hipótesis que señala diferencias significativas entre los cuatro exámenes. El análisis multivariante intrasujetos tampoco permite realizar alguna prueba post-hoc, como sucede cuando 
comparamos grupos. La razón de esto es que solo tenemos a un solo grupo y lo que se compara son sus diferencias. Para información de las personas interesadas en este procedimiento, vale la pena señalar que el algoritmo está ubicado dentro de la suite de herramientas del modelo lineal general (medidas repetidas) en el software estadístico SPSS.

TABLA 3

Análisis multivariante intrasujetos (Comparación entre exámenes)

\begin{tabular}{lll}
\hline Prueba de hipótesis & Valor del estadístico & p-valor \\
\hline Traza de Pillai & 0.516085029 & .000 \\
\hline Lambda de Wilks & 0.483914971 & .000 \\
\hline Traza de Hotelling & 1.066478741 & .000 \\
\hline Raíz mayor de Roy & 1.066478741 & .000 \\
\hline
\end{tabular}

Fuente: elaboración propia.

Nota: datos obtenidos en trabajo de campo.

Comparación entre medias y la calificación mínima aprobatoria. Con la finalidad de analizar con mayor profundidad los resultados, y tomando como valor de referencia la calificación de 6 (considerada institucionalmente como la calificación mínima aprobatoria), se ejecutó la prueba t de Student para una sola muestra. El resultado de este análisis estadístico señala que, únicamente en el examen 1 (nivel exploratorio), el estudiantado observado estuvo por debajo del 6 de calificación, mientras que en el resto de los exámenes la calificación media estadísticamente es aprobatoria. Lo anterior demuestra que la estrategia didáctica incrementó el aprendizaje estadístico aunque sea solo para alcanzar una calificación mínima aprobatoria. La Tabla 4 muestra la comparación de los promedios obtenidos en cada examen contra un valor de referencia, en este caso, 6 puntos.

TABLA 4

Comparación de calificaciones de exámenes contra el valor 6 t de Student para una sola muestra

\begin{tabular}{|c|c|c|c|}
\hline Criterio institucional de acreditación $=6$ & Calificación media grupal & $\mathrm{T}$ & GL p-valor \\
\hline Examen 1 & 4.3 & -5.880 & 32.000 \\
\hline Examen 2 & 5.6 & -1.811 & 32.080 \\
\hline Examen 3 & 6.3 & .966 & 32.341 \\
\hline Examen 4 & 5.6 & -1.222 & 32.231 \\
\hline
\end{tabular}

Fuente: elaboración propia.

Nota: datos obtenidos en trabajo de campo.

Evolución del aprendizaje estadístico. Por último, se presenta en la Figura 2 la evolución que tuvo el aprendizaje estadístico durante el semestre escolar. Cada examen, como ya se mencionó, incluyó aspectos teóricos y prácticos según cada nivel de investigación. De este modo, la calificación media y su error estándar del nivel exploratorio fue de $4.3 \pm 0.3$. Para el nivel descriptivo fue $5.6 \pm 0.2$, nivel relacional $6.3 \pm 0.4 \mathrm{y}$ nivel explicativo $5.6 \pm 0.4$. Así, se tiene entonces que el aprendizaje fue mejorando conforme avanzaba el semestre, a excepción de la última evaluación, en donde disminuyó. Aunque estadísticamente solo en el nivel exploratorio el grupo tuvo calificación reprobatoria, queda la insatisfacción con el aprendizaje logrado en el resto de los niveles investigativos. 


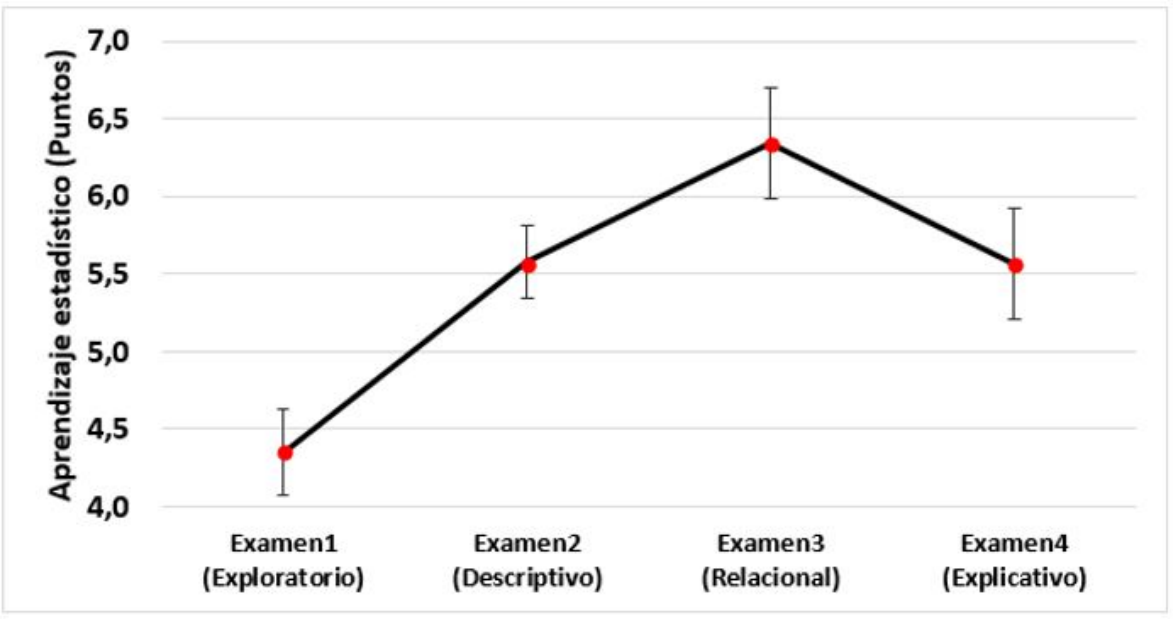

FIGURA 2.

Evolución del aprendizaje estadístico durante el semestre escolar. Un incremento en el aprendizaje estadístico fue constante en las tres primeras mediciones para decaer en la última.

Nota. El análisis multivariante (ANOVA de un factor intrasujetos) mostró diferencias significativas entre las cuatro mediciones. $(\mathrm{p}=0.000)$. La prueba W de Mauchly, demostró el supuesto de esfericidad que es un equivalente a la homogeneidad de varianzas cuando de grupos independientes se trata $(\mathrm{p}=0.173)$. Datos obtenidos en trabajo de campo. Fuente: elaboración propia

\section{Discusión}

Antes de dar inicio a la discusión de los resultados es conveniente mencionar que no se cuenta con registros del aprendizaje estadístico de nuestra población bajo situaciones diferentes a la que se presenta en este trabajo. En semestres académicos anteriores, por lo general no se le dio importancia al aprendizaje estadístico, lo que predominó fue el aprendizaje de la metodología de la investigación, por lo que los exámenes evaluaban otro tipo de competencias cognitivas y procedimentales. No fue sino hasta la publicación de los graves errores sobre la interpretación del valor p y el uso de las pruebas de hipótesis observados en 198 trabajos de investigación de estudiantes de Enfermería, que se decidió investigar con mayor profundidad la problemática y proponer algunas soluciones para mejorar la enseñanza de la Estadística (Alonso et al., 2018).

Según los resultados de esta investigación, el aprendizaje estadístico se incrementó conforme avanzó el semestre escolar a raíz de haber aplicado la estrategia educativa, sin embargo, académicamente se considera un efecto insuficiente. La evidencia estadística que se presenta, son los valores p de las cuatro pruebas de hipótesis que se desarrollaron con la ANOVA intrasujetos. Todas ellas arrojaron significancia estadística $(\mathrm{p} \leq 0.000)$, lo que significa que existen diferencias entre las calificaciones obtenidas en cada examen (Véase Tabla 3 y Figura 2). Dado que se trata de un análisis intrasujetos, no es factible realizar una prueba post-hoc, ya que no se tienen varios grupos a comparar, solo se cuenta con uno. Por esa razón, se realizó inmediatamente una prueba t de Student para una sola muestra (Tabla 4). De este modo se identificó que únicamente el examen 1 se puede considerar no aprobatorio, en consecuencia, la estrategia permite incrementar, por lo menos hasta 6 de calificación, el aprendizaje estadístico.

Si bien se observaron incrementos significativos entre la primera y las demás evaluaciones, ello no significa que el aprendizaje estadístico represente la adquisición de competencias cognitivas ni de habilidades procedimentales en Estadística. Cómo habría de serlo, si en el nivel exploratorio la calificación media fue de 4.3 puntos y solamente se incrementó 1.7 puntos para alcanzar 6 puntos en el resto de las evaluaciones. El único valor que podría otorgarse a este nivel de aprendizaje es que constituye un saber previo al nivel descriptivo. Es probable que los conocimientos previos del estudiantado respecto a los métodos que se aplican en el nivel exploratorio fueran escasos e incapaces de vincular con los nuevos conocimientos del nivel 
descriptivo. De acuerdo con la Teoría del aprendizaje significativo de Ausubel, lo que el alumnado sabe, el conocimiento previo, es un factor que influirá en los aprendizajes posteriores (Carretero, 1997). Deberá tomarse en cuenta que, cuando se implementaron los contenidos del nivel descriptivo, empezaron a figurar en la clase conceptos y procedimientos estadísticos univariados. Después, los procedimientos fueron bivariados y, por último, multivariados. Una posible explicación del decaimiento ocurrido en el examen cuatro es que el análisis multivariado es, en sí mismo, de mayor complejidad que el bivariado y univariado. Probablemente los conocimientos adquiridos en niveles inferiores fueron deficientes y no permitieron soportar procedimientos complejos de tipo multivariado; esto coincide con otras personas autoras, quienes han señalado dificultades en el aprendizaje y hasta verdaderos dolores de cabeza para lograr razonamientos estadísticos de cierta complejidad (Chance et al., 2004; Hernández et al., 2012; Inzunza y Jiménez, 2013; Maris y Aznar, 2018; Medina, 2014; Pulido, 2009).

Se debe reflexionar acerca de cuestiones como: ¿quiénes son las personas estudiantes?, ¿qué recursos tienen?, ¿cuáles son los apoyos institucionales?, ¿de cuánto tiempo disponen para estudiar?, ¿es el único curso de Estadística que han tomado? El estudiantado observado son personas que se encuentran al final de una formación académica orientada al cuidado de la salud de personas sanas y enfermas. El rol investigador que se menciona en el perfil de egreso debe permitir el desarrollo del pensamiento crítico y dejar atrás el pensamiento mágico o subjetivo que suele aflorar en una parte de profesionales de la Enfermería. Al respecto, Fawcett (2014) es clara y directa al señalar que, en Enfermería,

Cuando se lleva a cabo una investigación de caja negra, los hallazgos no constituyen teorías bien desarrolladas, las cuales son la mejor prueba de la práctica, sino que proporcionan evidencia para la práctica basada en creencias clínicas obstinadas, opiniones de las autoridades y el sentido común. (p. 291).

No se debe pretender formar una población estadística chiquita, sino más bien enfermeros y enfermeras que comprendan el contenido de los artículos científicos, que puedan realizar una investigación con calidad metodológica y estadística aceptable, que puedan enfrentar un examen de ingreso al posgrado con buenas notas y que, como parte de la ciudadanía del siglo XXI, desarrollen su criterio científico para la toma de decisiones razonadas y dejen a un lado explicaciones determinísticas de eventos, en los cuales los resultados aleatorios son la regla y no la excepción. En la Universidad se dispone de los recursos para lograr tal objetivo, pues se cuenta con laboratorios de computación, Internet, software estadístico y material bibliográfico, y una plantilla de profesores y profesoras con estudios de posgrado que son evaluados anualmente respecto a su productividad por la institución educativa y respecto a su desempeño académico por el mismo estudiantado.

Para el caso específico del aprendizaje estadístico, las políticas institucionales, desafortunadamente, no exigen como prerrequisito el manejo y dominio del software estadístico, ni tampoco se incluye una formación curricular al respecto (Facultad de Estudios Superiores Iztacala, UNAM, 2013). Probablemente, la raíz del problema se ubique en su formación académica en el nivel básico y medio superior, la cual, de acuerdo con estándares internacionales, ha demostrado que deja mucho que desear en las áreas de Matemáticas y Ciencias (Salinas et al., 2018).

No obstante, como ya ha sido analizado por otras personas autoras, quizás los factores actitudinal y socioeconómico tengan un impacto negativo en el estudiantado y sea algo sobre lo que hay que trabajar con mayor ahínco, sin perder de vista la ayuda que puedan brindar las tutorías y otros apoyos institucionales en materia de becas de diversa índole (Alonso, 2015; Alonso et al., 2015; Perrenoud, 2011; Rojas-Betancur y Méndez-Villamizar, 2013).

El tiempo disponible para el aprendizaje de la Estadística representa una limitación muy importante. Si se considera que, además del aspecto estadístico, el programa del curso incluye el desarrollo de un proyecto de investigación, diseño, construcción y validación de instrumentos de medición, trabajo de campo, elaboración del informe final, falta de exclusividad de los laboratorios de computación y dificultades intrínsecas de la materia, en definitiva, el tiempo es un factor en contra de la didáctica del curso. 
En la licenciatura en Enfermería, el único acercamiento que el estudiantado tiene hacia la estadística es cuando cursan la materia Métodos Cuantitativos de Investigación (Facultad de Estudios Superiores Iztacala. UNAM, 2014; Alonso et al., 2018; Alonso, 2012). Esta situación implica un gran reto para el profesorado, pues también ha sido documentado que, desde el nivel básico hasta el superior, la formación académica especializada en Matemáticas, en especial en Estadística, es heterogénea. Al respecto, algunas entidades autoras han reportado la mirada del estudiantado al aprender estos contenidos, en donde predomina la versión de que un factor que limita el aprendizaje es el profesorado mismo (Cademartori y Grimaldi, 2019).

El contexto de aplicación de los aprendizajes adquiridos por parte del estudiantado de Enfermería, desafortunadamente en la mayoría de los casos, se limita al análisis estadístico de su proyecto semestral, de sus tesis para titulación y como elemento importante para ser admitido en un posgrado. En la práctica profesional, es de dominio público que una gran mayoría de la población egresada se desempeñará en el área asistencial. La proporción de profesionales de la Enfermería que alcanzarán una plaza en investigación, tal y como ocurre en la mayoría de las profesiones, es muy limitada. Por esa razón, sería mejor enfocar los esfuerzos docentes para lograr dos propósitos encadenados y secuenciados en el tiempo; primero, lograr el mejor aprendizaje estadístico posible e inmediatamente después lograr formar ciudadanos con cultura estadística. Esto significa interrelacionar dos componentes: a) capacidad para interpretar y evaluar críticamente la información estadística, y b) capacidad para discutir o comunicar opiniones respecto a tales informaciones estadísticas cuando sea relevante (Arteaga et al., 2011). Se ha enfatizado la importancia del desarrollo del razonamiento estadístico en una sociedad caracterizada por la disponibilidad de una gran cantidad de datos e información (Batanero, 2002).

También, se defiende la idea de que el Aprendizaje Estadístico juega o debe jugar un papel primordial en el desarrollo de la sociedad actual, ya que proporciona las herramientas para describir y analizar situaciones en donde la variabilidad y la incertidumbre coexisten y hay que tomar decisiones razonadas (Ponteville, 2014). Después de haber reflexionado acerca de la utilidad del aprendizaje estadístico, se defiende la postura de que el profesorado debe promover constantemente una actitud positiva, diseñar y volver a diseñar estrategias educativas con el ideal de colaborar en la formación, no de personas enfermeras estadísticas o estadísticas aficionadas, sino de ciudadanos y ciudadanas capaces de observar fenómenos aleatorios, reconocer la variabilidad y la incertidumbre inherentes, plantearse razonamientos a través de datos, con los que generen información y tomen las mejores decisiones posibles. El debate permanente entre el personal docente acerca de los resultados desalentadores en el aprendizaje estadístico (como ocurrió en este caso) debe ser la semilla de más y mejor investigación educativa. No obstante, se sostiene la tesis de continuar investigando sobre cómo lograr mejores resultados con la estrategia propuesta y no regresar a la estrategia de enseñanza tradicional que, a grandes rasgos, solo logró aversión a la Estadística y fueron extremadamente escasas las calificaciones mayores a 7 puntos. Con la estrategia propuesta hubo casos de estudiantes con calificaciones de 8.7 y 8.8 puntos, lo que según Hilde (2021) se explica por la diversidad de capacidades cognitivas y personalidades heterogéneas que a veces son compatibles con algunas estrategias de enseñanza, pero que en otras ocasiones les desfavorece, como podría haber ocurrido con el estudiantado que obtuvo muy bajas calificaciones en cada uno de los exámenes.

\section{Conclusiones}

La estrategia educativa orientada al mejoramiento del aprendizaje estadístico tuvo el efecto esperado desde un enfoque estadístico, en el sentido de que, al comparar la primera calificación con las demás, la calificación media se incrementó significativamente en 1.7 puntos; pasó de 4.3 a 6 puntos. Sin embargo, desde la perspectiva educativa, las calificaciones medias alcanzadas representan puntajes deficientes o, en el mejor de los casos, mínimo aprobatorio, de acuerdo a la escala de calificación que se utiliza en México. Cabe destacar que el rango de calificaciones obtenidas en los exámenes permite observar que, en algunos casos, 
hubo un muy bajo nivel de aprendizaje; en contraste, en otros casos se demostró un alto nivel de aprendizaje estadístico. Lo anterior podría significar que existen determinantes personales (como la capacidad cognitiva o personalidad), sociales (como el nivel socioeconómico) e institucionales (como la formación de sus docentes) que probablemente influyen en el rendimiento académico del estudiantado de la licenciatura en Enfermería.

\section{ReFERENCIAS}

Alonso, J. (2012). Evolución y enseñanza de la investigación en Enfermería. Vinculación epidemiológica y estadística. Editorial Académica Española.

Alonso, J. (2015). Actitud hacia la investigación y logro escolar durante el aprendizaje de la Estadística de estudiantes de Enfermería. Revista Cuidarte, El arte del cuidado, 4(8), 8-16. https://doi.org/10.22201/fesi.23958979e.20 15.4.8.69096

Alonso, J. y Alonso, A. (2019). Investigación cientifica en Enfermería. Análisis de datos. Editorial de la Facultad de Estudios Superiores Iztacala, Universidad Nacional Autónoma de México.

Alonso, J., Alonso, A., Ordoñez, A y Cruz, L. (2018). Methodological quality on academic research reports from nursing students. International Educational Applied Scientific Research Journal, 3(5), 3-6. http://ieasrj.com/jou $\mathrm{rnal} / \mathrm{index} \cdot \mathrm{php} / \mathrm{ieasrj} / \mathrm{article} / \mathrm{view} / 100$

Alonso, J., Alonso, A. y Valadez, D. (2015). Actitud hacia la investigación científica de estudiantes de Enfermería. Revista Cuidarte, El arte del cuidado, 4(7), 24-35. https://doi.org/10.22201/fesi.23958979e.2015.4.7.69106

Alonso, J., Alonso, A., Zamora, F., Medina, C. (2017). Aprender haciendo; modelos predictivos en la enseñanza y aprendizaje de estadística. Social Science Learning Education Journal, 2(10). http://www.sslej.in/index.php/ssl ej/article/view/1459

Alonso, J., Cuevas, L. y Alonso, A. (2020). Diagnóstico sobre competencias en investigación de estudiantes de enfermería. Revista Cuidarte "El arte del cuidado", 9(17), 6-18. http://dx.doi.org/10.22201/fesi.23958979e.20 20.9.17.72755

Arteaga, P., Batanero, C., Cañadas, C. y Contreras, M. (2011). Las tablas y gráficos estadísticos como objetos culturales. Números. Revista de didáctica de las matemáticas, 76, 55-67. https://www.academia.edu/26336038/Las_Tabla s_y_Gr\%C3\%A1ficos_Estad\%C3\%ADsticos_como_Objetos_Culturales

Batanero, C. (2001) Didáctica de la Estadística. Departamento de didáctica de la matemática. Universidad de Granada. https://www.researchgate.net/publication/255738320_Didactica_de_la_Estadistica

Batanero, C. (2002). Los retos de la cultura estadística. Jornadas Interamericanas de Enseñanza de la Estadística. http: //www.ugr.es/ batanero/pages/ARTICULOS/CULTURA.pdf

Cademartori, P. y Grimaldi, V. (2019). Explicar bien, aprender bien. La relación con el saber en las voces de los alumnos. Yupana, (11), 58-74. https://doi.org/10.14409/yu.v0i11.8842

Carretero, M. (1997). ¿Qué es el constructivismo? En M. Carretero (Ed.). Desarrollo cognitivo y aprendizaje. Constructivismo y educación. (pp. 39-71). Progreso https://www.researchgate.net/profile/Cesar-Coll-2/publication/48137926_Que_es_el_constructivi smo/links/53eb30a20cf2fb1b9b6afb55/Que-es-el-constructivismo.pdf

Chance, B., Del Mas, R. y Garfield, J. (2004). Reasoning about sampling distributions. En D. Ben-Zvi y J. Garfield (Eds.), The challenge of developing statistical literacy, reasoning, and thinking. Kluwer Academic Publishers. Purdue University.

Díaz-Barriga, F. (2006). Enseñanza situada: Vinculo entre la escuela y la vida. McGraw-Hill.

Facultad de Estudios Superiores Iztacala, UNAM. (2013). Perfil de Ingreso y Egreso. Carrera de Enfermería. http://e nfermeria.iztacala.unam.mx/enf_perfiles.php

Facultad de Estudios Superiores Iztacala, UNAM. (2014). Plan de estudios. Carrera de Enfermería. http://enfermeri a.iztacala.unam.mx/enf_planest.php 
Fawcett, J. (2014). Tendencias de investigación en enfermería. Aquichan, 14(3), 289-293. http://dx.doi.org/10.529 4/aqui.2014.14.3.1

Ferreri, N., Panzeri, A., Santone, B., Storza, M., De Giorgio, N., Elchaimi, M., Álvarez, E. y Molina, G. (1999, octubre). Enseñanza de la Estadística: Desde el ámbito laboral al aula. Memorias de las Cuartas Jornadas "Investigaciones en la Facultad" de Ciencias Económicas y Estadística", Rosario Argentina. https://www.fcecon.unr.edu.ar/web/site s/default/files/u16/Decimocuartas/Ferreri,Panzeri,Santone_ensenanza\%20de\%20la\%20estadistica.PDF

Garbanzo, G (2007). Factores asociados al rendimiento académico en estudiantes universitarios, una reflexión desde la calidad de la educación superior pública. Revista Educación, 31(1), 43-63. https://www.redalyc.org/articulo .oa? id $=44031103$

Hernández, L., González, C., González, V. y Rodríguez, M. (2012). Acercamiento al proceso de enseñanza aprendizaje de estadística en el currículo de Medicina. Revista Cubana de Informática Médica, 4(2), 184-189. http://scielo. sld.cu/scielo.php?script=sci_arttext\&pid=S1684-18592012000200008\&lng=es\&tlng=es

Hilde, V., Helge, B., Trygve, A., Kathrine, F., y Solve, S. (2021). Adapting statistics education to a cognitively heterogeneous student population. Journal of Statistics and Data Science Education, 29(2), 183-191. https://do i.org/10.1080/26939169.2021.1928573

Inzunza, S. y Jiménez, V. (2013). Caracterización del razonamiento estadístico de estudiantes universitarios acerca de las pruebas de hipótesis. Revista latinoamericana de investigación en Matemática Educativa, 16(2), 179-211. ht tps://doi.org/10.12802/relime.13.1622

Maris, S. y Aznar, A. (2018). Razonamiento Estadístico en Estudiantes de Ingeniería. Yupana, 18(12), 23-39. https ://doi.org/10.14409/yu.v0i12.9625

Medina, I. (2014). Competencias en investigación en estudiantes de la licenciatura en Enfermería de la FES-I UNAM. [Tesis de licenciatura], Universidad Nacional Autónoma de México. México.

National Council of Teachers of Mathematics [NCTM]. (2014). De los principios a la acción. Editando libros. https://www.nctm.org/uploadedFiles/Standards_and_Positions/Principles_to_Actions/Principles\%20 to\%20Actions\%20Executive\%20Summary\%20(Spanish).pdf

Orosz, T. (2016). Online versus on-ground: student outcomes and the influence of student engagement in a college success course [Tesis de doctorado]. Rowan University https://rdw.rowan.edu/cgi/viewcontent.cgi?referer=\&httpsred ir $=1 \&$ article $=3343 \&$ context $=$ etd

Ortiz, D (2015). El constructivismo como teoría y método de enseñanza. Sophia, Colección de Filosofía de la Educación, (19), 93-110 https://www.redalyc.org/articulo.oa?id=441846096005

Perrenoud, P. (2011). Construir competencias desde la escuela. Juan Carlos Sáez editor.

Ponteville, C. (2014). ¿Para qué enseñamos Estadística? En P. Lestón (Ed.), Acta Latinoamericana de Matemática Educativa (pp. 517-525). http://funes.uniandes.edu.co/5450/1/PontevilleParaqueALME2014.pdf

Posner, G. (1993). Field experience. A Guide to Reflective Thinking. (3a ed.). Longman Pub Group.

Prieto, L. (2006). Aprendizaje activo en el aula universitaria: el caso del aprendizaje basado en problemas, en Miscelánea Comillas. Revista de Ciencias Humanas y Sociales 64(124), 173-196. https://revistas.comillas.edu/index.php/m iscelaneacomillas/article/view/6558/6367

Pulido, E. (2009). Enseñanza de la estadística a partir de la actitud del alumno. Laurus, 15(30), 42-70. https://www .redalyc.org/pdf/761/76120651003.pdf

Rojas-Betancur, M. y Méndez-Villamizar, R. (2013). Cómo enseñar a investigar. Un reto para la pedagogía universitaria. Educación y Educadores, 16(1), 95-108. https://www.redalyc.org/pdf/834/83428614001.pdf

Salinas, D., De Moraes, C. y Schwabe, M. (2018). Programa para la Evaluación Internacional de Alumnos de la OCDE. PISA 2018. https://www.oecd.org/pisa/publications/PISA2018_CN_MEX_Spanish.pdf

Supo, J. (2014) Seminarios de investigación cientifica. Metodología de la investigación para las ciencias de la salud. BIOESTADISTICO EIRL.

Universidad Nacional Autónoma de México. (1997). Normatividad administrativa de la UNAM. Reglamento General de Exámenes. UNAM. http://www.ddu.unam.mx/index.php/reglamento-general-de-examenes 
Universidad Nacional Autónoma de México. (2015). Proyecto de modificación del plan de estudios de la licenciatura en Enfermería. Tomo II. https://enfermeria.iztacala.unam.mx/documentos/Tomos_Plan_Estudios_Lic/Tomo_I I_Enfermeria-FESI.pdf

\section{INFORMACIÓN ADICIONAL}

Cómo citar: Alonso-Trujillo, J., Alonso-Ricardez, A., Valera-Mota, M. M. y Cuevas-Guajardo, L. (2022). Aprendizaje estadístico basado en niveles de investigación. Revista Educación, 46(1). http:// doi.org/10.15517/revedu.v46i1.45425 